\title{
A projection-based TODIM method under multi-valued neutrosophic environments and its application in personnel selection
}

\author{
Pu Ji ${ }^{1} \cdot$ Hong-yu Zhang ${ }^{1} \cdot$ Jian-qiang Wang ${ }^{1}$
}

Received: 10 April 2016/Accepted: 8 June 2016

(C) The Natural Computing Applications Forum 2016

\begin{abstract}
The personnel selection is a vital activity for companies, and multi-valued neutrosophic sets (MVNSs) can denote the fuzziness and hesitancy in the processes of the personnel selection. The extant fuzzy TODIM (an acronym in Portuguese of interactive and multi-criteria decision-making) methods take advantage of distance to denote the difference between two fuzzy sets (FSs). Nevertheless, the distance measurement, which ignores the included angle between two FSs, cannot comprehensively reflect the difference between two FSs. To cover this defect, a projection-based TODIM method with MVNSs for personnel selection is established to consider the risk preference of decision-makers and overcome the defect of the extant fuzzy TODIM methods. The proposed TODIM method makes use of an improved comparison method which overcomes the deficiency of extant comparison method. Furthermore, a projection-based difference measurement is defined and utilized in the projection-based TODIM method. We conduct a numerical example of the personnel selection to explain the application of the projection-based TODIM method and discuss the influence of the parameter. Finally, the proposed method is compared with several extant methods to verify its feasibility.
\end{abstract}

Keywords Multi-criteria decision-making · Multi-valued neutrosophic sets · Projection · TODIM method .

The personnel selection

Jian-qiang Wang

jqwang@csu.edu.cn

1 School of Business, Central South University, Changsha 410083, China

\section{Introduction}

Personnel selection is an important issue for companies due to its great influence on the development of companies. Many researchers have utilized multi-criteria decisionmaking (MCDM) methods to tackle practical personnel selection problems [1-4]. Some of these researchers pointed out that fuzzy, uncertain and incomplete information exist in the processes of personnel selection [5, 6]. To deal with this kind of information, they introduced the fuzzy logic and fuzzy sets (FSs). FSs were originally defined by Zadeh [7], and it has been greatly extended [8, 9]. For instance, Atanassov [10] proposed intuitionistic fuzzy sets (IFSs). To express uncertainty, Atanassov and Gargov [11] extended IFSs and presented interval valued intuitionistic fuzzy sets (IVIFSs). In some situations, hesitancy may exist when decision-makers determine the membership degree of an object. To depict this hesitant information, Torra [12] developed hesitant fuzzy sets (HFSs). Furthermore, Smarandache [13, 14] defined neutrosophic sets (NSs) to reflect the truth, indeterminate and false information simultaneously. Additionally, Wang et al. [15] pointed out that NSs were difficult to apply in practical problems. To overcome this defect, they proposed single-valued neutrosophic sets (SVNSs) [15]. Moreover, some other extensions of FSs have been developed [16, 17], including simplified NSs [18, 19] and interval valued NSs [20-22], and combined with other theories, like graph theory [23-27] and prospect theory [28]. In addition, the application of sundry extensions of FSs has been studied by researchers in a variety of fields, like decision-making [29-33], medical service [34, 35], cloud service selection [36] and the supplier selection [37, 38].

Multi-valued neutrosophic sets (MVNSs), as the integration of HFSs and SVNSs, have drawn researchers' 
attention. MVNSs were initially defined by Wang and $\mathrm{Li}$ [39]. They [39] made use of three sets, each of which is composed of different real numbers in $[0,1]$, to characterize the degrees of truth-membership, indeterminacy-membership and falsity-membership, respectively. MVNSs, which combine the merits of HFSs and SVNSs, can describe fuzzy information in more detail than FSs, IFSs, HFSs and SVNSs. Furthermore, MVNSs have been applied in MCDM problems. For example, Peng et al. [40] defined two weighted power aggregation operators and established a decision-making method with these two operators. Ye [41] called MVNSs single-valued neutrosophic hesitant fuzzy sets and constructed two multi-valued neutrosophic MCDM methods with the proposed multi-valued neutrosophic weighted averaging (MVNWA) and multi-valued neutrosophic weighted geometric (MVNWG) operators. Besides the operators, the correlation coefficient of MVNSs was proposed. Sahin and Liu [42] established a novel MCDM method based on the proposed correlation coefficient. What is more, Liu and Zhang [43] presented the distance measurement between MVNSs and applied the VIKOR (VlseKriterijumska Optimizacija I Kompromisno Resenje) method to address MCDM problems under multi-valued neutrosophic environments. In addition, the comparison method for MVNSs was defined by Liu and Zhang [43].

MVNSs are the perfect means to represent fuzzy information in the personnel selection processes. For example, when the interviewer of a company evaluates a candidate, he or she may hesitate about the degree to which he or she thinks the candidate is capable for the position. He or she may prefer to depict this kind of hesitant information with several real numbers between 0 and 1 rather than one single real number, such as $\{0.6,0.7,0.8\}$. Moreover, there is hesitancy in the degree to which he or she thinks the candidate is not competent for the position, such as he or she describes the degree of falsity-membership as $\{0.1,0.2\}$. Additionally, the degree to which he or she is not sure if the candidate is qualified for the position is a set of several real numbers within $[0,1]$, such as $\{0.2,0.3\}$. As illustrated above, a personnel selection problem may include truth-membership, indeterminacy-membership and falsity-membership degrees at the same time, and each of these three degrees of membership may be a set of several different real numbers between 0 and 1 . Therefore, MVNSs are more effective in describing fuzzy and hesitant information in personnel selection problems than FSs, IFSs, HFSs and SVNSs.

The projection measurement is a significant tool in MCDM. It depicts both the distance and the included angle between two elements. Compared with the distance measurement, the projection measurement can reflect the difference between two elements more exactly. The projection measurement has been extended into many kinds of fuzzy environments [44, 45]. For instance, $\mathrm{Xu}$ and $\mathrm{Hu}$ [46] extended projection measurements into intuitionistic fuzzy and interval intuitionistic fuzzy environments and constructed MCDM methods based on the proposed projection measurements. Furthermore, Zhang et al. [47] introduced the intuitionistic trapezoidal fuzzy projection measurement and developed a gray rational projection method for MCDM problems.

To consider the risk preferences of decision-makers, TODIM (An acronym in Portuguese of interactive and decision-making method named Tomada de decisao interativa e multicritévio) method was developed by Gomes and Lima [48, 49] on the basis of the prospect theory [50]. To tackle fuzzy MCDM problems, Krohling and Souza [51] defined the fuzzy TODIM method based on the TODIM method in Refs. [48, 49]. Since then, the fuzzy TODIM methods under various fuzzy environments have been researched and applied to settle MCDM problems [52-54]. For instance, Tseng et al. [55] utilized TODIM method to solve MCDM in the evaluation of green supply chain practices under triangular fuzzy environments. In addition, TODIM methods under intuitionistic fuzzy and interval intuitionistic fuzzy environments were established by Lourenzutti and Krohling [56] and Li et al. [57]. Zhang and $\mathrm{Xu}$ [58] introduced the fuzzy TODIM method to address hesitant fuzzy MCDM problems. Moreover, TODIM method with neutrosophic numbers was proposed and applied in decision-making by Zhang et al. [59].

MVNSs can be used to express the fuzzy and hesitant information in the processes of personnel selection. Furthermore, the extant comparison method of MVNSs has some deficiencies that will be illustrated in Sect. 2.1. Moreover, the distance measurement ignores the included angle between objects while the projection measurement considers the included angle between elements besides the distance. In other words, the projection measurement can depict difference between objects more exactly than the distance measurement. However, the extant TODIM methods utilize the distance measurement to depict the difference between objects. Motivated by these, we established a projection-based TODIM method to solve the personnel selection problems under multi-valued neutrosophic environments. To do that, an improved comparison method was defined to overcome the deficiency of the extant comparison method. Then, we presented the projection and normalized projection measurements of MVNSs. Subsequently, a projection-based difference measurement was defined to denote the difference between two MVNSs. In the proposed personnel selection method, the projection-based difference measurement was incorporated with the fuzzy TODIM method to cover the shortcoming of the extant fuzzy TODIM methods. In addition, the improved comparison method was utilized in 
the projection-based TODIM method to judge whether there is a gain, loss or breakeven. The projection-based TODIM method was proven to be capable of successfully tackling the personnel selection problems by a numerical example. What is more, a comparative analysis certified the feasibility of the projection-based TODIM method.

The structure of this paper is organized as follows. Section 2 presents several related notions of MVNSs and the fuzzy TODIM method. We define an improved comparison method, the projection and normalized projection measurements, and the projection-based difference measurement of MVNSs in Sect. 3. Moreover, a projectionbased TODIM method is constructed in Sect. 3. In Sect. 4, the application of the proposed method is illustrated by a numerical example of personnel selection. Furthermore, we study the influence of the parameter in Sect. 4. A comparative analysis is also provided and discussed in Sect. 4. Finally, in Sect. 5, we conclude the paper and provide some directions for future research.

\section{Preliminaries}

This section reviews some basic concepts of MVNSs and fuzzy TODIM method. And these concepts will be utilized in the reminder of this study.

\subsection{MVNSs}

Definition 1 [39] Let $X=\left\{x_{1}, x_{2}, \ldots, x_{n}\right\}$ be a non-empty fixed set. An MVNS can be defined as:

$A=\left\{x_{t},\left(\tilde{t}_{A}\left(x_{t}\right), \tilde{i}_{A}\left(x_{t}\right), \tilde{f}_{A}\left(x_{t}\right)\right)\right\}$,

where $x_{t} \in X$ and $\tilde{t}_{A}\left(x_{t}\right), \tilde{i}_{A}\left(x_{t}\right)$ and $\tilde{f}_{A}\left(x_{t}\right)$ are three sets each of which is composed of different values in $[0,1]$. Let $l_{t}, l_{i}$ and $l_{f}$ denote the numbers of elements in $\tilde{t}_{A}\left(x_{t}\right), \tilde{i}_{A}\left(x_{t}\right)$ and $\tilde{f}_{A}\left(x_{t}\right)$, respectively. $\tilde{t}_{A}\left(x_{t}\right)=\left\{t_{A 1}\left(x_{t}\right), t_{A 2}\left(x_{t}\right), \ldots, t_{A l_{t}}\left(x_{t}\right)\right\}$ is a set comprising all possible truth-membership degrees, $\tilde{i}_{A}\left(x_{t}\right)=\left\{i_{A 1}\left(x_{t}\right), i_{A 2}\left(x_{t}\right), \ldots, i_{A l_{i}}\left(x_{t}\right)\right\}$ is a set comprising all possible indeterminacy-membership degrees and $\tilde{f}_{A}\left(x_{t}\right)=$ $\left\{f_{A 1}\left(x_{t}\right), f_{A 2}\left(x_{t}\right), \ldots, f_{A l_{f}}\left(x_{t}\right)\right\}$ is a set comprising all possible falsity-membership degrees. We assume that elements in $\tilde{t}_{A}\left(x_{t}\right), \tilde{i}_{A}\left(x_{t}\right)$ and $\tilde{f}_{A}\left(x_{t}\right)$ are in increasing order in this paper for the ease of narration.

In addition, $\left\{\tilde{t}_{A}\left(x_{t}\right), \tilde{i}_{A}\left(x_{t}\right), \tilde{f}_{A}\left(x_{t}\right)\right\}$, which is an element in $A$, is a multi-valued neutrosophic number (MVNN). For convenience, an MVNN is denoted by $a=\left\{\tilde{t}_{A}, \tilde{i}_{A}, \tilde{f}_{A}\right\}$.

Definition 2 [43] Let $b=\left\{\tilde{t}_{B}, \tilde{i}_{B}, \tilde{f}_{B}\right\}$ be an MVNN. The score function of $b$ can be defined as: $s(b)=\left(\frac{1}{l_{t}} \sum_{k=1}^{l_{t}} t_{B_{k}}+\frac{1}{l_{i}} \sum_{g=1}^{l_{i}}\left(1-i_{B_{g}}\right)+\frac{1}{l_{f}} \sum_{r=1}^{l_{f}}\left(1-f_{B_{r}}\right)\right) / 3$.

Definition 3 [43] Let $b$ and $c$ be two MVNNs. The comparison method between $b$ and $c$ can be defined as:

1. When $s(b)<s(c), b \prec c$;

2. When $s(b)>s(c), b \succ c$; and

3. When $s(b)=s(c), b=c$.

Nevertheless, a limitation exists in the comparison method in Definition 3. The following example depicts this limitation.

Example 1 Let $b=\{\{0.3,0.4,0.5\},\{0.2,0.3\},\{0.1\}\}$ and $c=\{\{0.2,0.4,0.6\},\{0.1,0.2\},\{0.2\}\} \quad$ be two MVNNs. It is evident that $b \neq c$. By Definition 2, $s(b)=((0.3+0.4+0.5) / 3+(1-0.2+1-0.3) / 2+$ $(1-0.1) / 3)=0.683$ and $s(c)=((0.2+0.4+0.6) / 3+$ $(0.9+0.8) / 2+(1-0.2) / 3)=0.683$. That is to say, $s(b)=s(c)$. By the comparison method in Definition 3, $b=c$, which is against our intuition.

\subsection{The fuzzy TODIM method}

Krohling and Souza [51] presented a fuzzy TODIM method considering that the traditional TODIM method cannot address problems under fuzzy environments. The details of the fuzzy TODIM method are introduced in the rest of this subsection.

Let us consider an MCDM problem with $m$ alternatives $A=\left\{A_{1}, A_{2}, \ldots, A_{m}\right\}$, and $n$ criteria $C=\left\{C_{1}, C_{2}, \ldots C_{n}\right\}$. The decision-making matrix can be denoted as:

$S=\left(\begin{array}{cccc}S_{11} & S_{12} & \cdots & S_{1 n} \\ S_{21} & S_{22} & \cdots & S_{2 n} \\ \vdots & \vdots & \ddots & \vdots \\ S_{m 1} & S_{m 2} & \cdots & S_{m n}\end{array}\right)$,

where $S_{t j}$ is a trapezoidal fuzzy number and it represents the value of alternative $A_{t}$ under criterion $C_{j}$. Furthermore, the weight vector of criteria is $w=\left(w_{1}, w_{2}, \ldots, w_{n}\right)^{T}$, where $w_{j} \geq 0(j=1,2, \ldots, n)$ and $\sum_{j=1}^{n} w_{j}=1$.

Step 1 Normalize the decision matrix.

Step 2 Obtain the dominance of each alternative $A_{i}$ over each alternative $A_{r}$.

$\Phi_{i r}=\sum_{j=1}^{n} \Phi_{i r}^{j}$ 
where

$$
\Phi_{i r}^{j}=\left\{\begin{array}{ll}
\sqrt{\frac{w_{j u}}{\sum_{j=1}^{n} w_{j u}}} \cdot d\left(S_{i j}, S_{r j}\right), & \text { if }\left(m\left(S_{i j}\right)-m\left(S_{r j}\right)\right)>0 \\
0, & \text { if }\left(m\left(S_{i j}\right)-m\left(S_{r j}\right)\right)=0 \\
\frac{-1}{t} \sqrt{\frac{\sum_{j=1}^{n} w_{j u}}{w_{j u}}} \cdot d\left(S_{i j}, S_{r j}\right), & \text { if }\left(m\left(S_{i j}\right)-m\left(S_{r j}\right)\right)<0
\end{array} .\right.
$$

$w_{j u}=\frac{w_{j}}{w_{u}} \quad$ and $\quad w_{u}=\max \left(w_{j}\right)(j=1,2, \ldots, n) . \quad d\left(S_{i j}, S_{r j}\right)$ signifies the distance between $S_{i j}$ and $S_{r j} \cdot m\left(S_{i j}\right)$ is the defuzzified value defined in Ref. [51]. Here, $m\left(S_{i j}\right)$ is utilized to compare two fuzzy numbers. If $\left(m\left(S_{i j}\right)-\right.$ $\left.m\left(S_{r j}\right)\right)>0$, it represents a gain; if $\left(m\left(S_{i j}\right)-m\left(S_{r j}\right)\right)=0$, it is nil; if $\left(m\left(S_{i j}\right)-m\left(S_{r j}\right)\right)<0$, it represents a loss. The parameter $t$ is the decay factor of the loss and $t>0$ under normal circumstances.

Step 3 Obtain the global value of the alternative $A_{i}$ via the following formula:

$\xi_{i}=\frac{\sum_{r=1}^{m} \Phi_{i r}-\min _{1 \leq i \leq m}\left(\sum_{r=1}^{m} \Phi_{i r}\right)}{\max _{1 \leq i \leq m}\left(\sum_{r=1}^{m} \Phi_{i r}\right)-\min _{1 \leq i \leq m}\left(\sum_{r=1}^{m} \Phi_{i r}\right)}$.

The rank order of the alternatives can be obtained according to the global value of each alternative. The smaller the global value $\xi_{i}$, the worse the alternative $A_{i}$ will be.

A shortcoming exists in this fuzzy TODIM method. In Eq. (1), the distance measurement is utilized to reflect the difference between two fuzzy numbers. However, the difference between two fuzzy numbers includes both the distance and the included angle between them while the distance measurement ignores the latter. In other words, the distance measurement in this fuzzy TODIM method cannot reflect the difference between two fuzzy numbers fully and accurately.

\section{A new extended TODIM method for MCDM problems with MVNSs}

In this section, a new comparison method of MVNSs is defined to overcome the limitation presented in Sect. 2.1. Moreover, we propose the projection and normalized projection measurements of MVNSs. Subsequently, based on these two measurements, a projection-based difference measurement is presented. Finally, we construct a new projection-based TODIM method on the basis of the projection-based difference measurement.

\subsection{A new comparison method of MVNNs}

Definition 4 Let $b=\left\{\tilde{t}_{B}, \tilde{i}_{B}, \tilde{f}_{B}\right\}$ be an MVNN. The score function for $b$ can be defined as:

$s(b)=\left(3+\frac{1}{l_{t}} \sum_{k=1}^{l_{t}} t_{B k}-\frac{2}{l_{i}} \sum_{g=1}^{l_{i}} i_{B g}-\frac{1}{l_{f}} \sum_{r=1}^{l_{f}} f_{B r}\right) / 4$

and the accuracy function for $b$ can be defined as:

$$
\begin{aligned}
& h(b)= \\
& \left(3+\frac{1}{l_{t}} \sum_{k=1}^{l_{t}} t_{B k}-\frac{2}{l_{i} l_{t}} \sum_{g=1}^{l_{i}} \sum_{k=1}^{l_{t}} i_{B g}\left(1-t_{B k}\right)-\frac{1}{l_{f} l_{i}} \sum_{r=1}^{l_{f}} \sum_{g=1}^{l_{i}} f_{B r}\left(1-i_{B g}\right)\right) / 4 .
\end{aligned}
$$

Definition 5 Let $b$ and $c$ be two MVNNs. The comparison method between $b$ and $c$ can be defined as:

1. When $s(b)<s(c), b \prec c$;

2. When $s(b)>s(c), b \succ c$;

3. When $s(b)=s(c)$ and $h(b)<h(c), b \prec c$;

4. When $s(b)=s(c)$ and $h(b)>h(c), b \succ c$; and

5. When $s(b)=s(c)$ and $h(b)=h(c), b=c$.

Example 2 Use the data in Example 1. By Eq. (2), we have that $s(b)=(3+(0.3+0.4+0.5) / 3-(0.2+0.3)-$ $0.1) / 4=0.7, \quad s(c)=(3+(0.2+0.4+0.6) / 3-(0.1+$ $0.2)-0.2) / 4=0.975$. Therefore, $s(b)<s(c)$. By Definition 5 , we can obtain that $b \prec c$, that is, the comparison method in Definition 5 conquers the defect of the comparison method in Definition 3.

Example 3 Let $b=\{\{0.3,0.4,0.5\},\{0.2,0.3\},\{0.1\}\}$ and $c=\{\{0.4,0.5,0.6\} \cdot\{0.2,0.3\},\{0.2\}\}$ be two MVNNs. By Eq. (2), we have that $s(b)=0.7$ and $s(c)=0.7$. By Eq. (3), $h(b)=0.756$ and $h(c)=0.775$. Hence, $s(b)=s(c)$ and $h(b)<h(c)$. By Definition 5, we can obtain that $b \prec c$.

Definition 6 Let $b=\left\{\tilde{t}_{B}, \tilde{i}_{B}, \tilde{f}_{B}\right\}$ be an MVNN. The complementary set neg $(b)$ of $b$ can be defined as:

$\operatorname{neg}(b)=\left\{\tilde{f}_{B}, \tilde{i}_{\text {neg }(B)}, \tilde{t}_{B}\right\}$,

where $\tilde{i}_{\text {neg }(B)}=\left\{1-t_{B l_{i}}\left(x_{t}\right), 1-t_{B\left(l_{i}-1\right)}\left(x_{t}\right), \ldots, 1-i_{B 1}\right.$ $\left.\left(x_{t}\right)\right\}$.

\subsection{The projection and normalized projection measurements of MVNSs}

In this subsection, we define a cosine measurement of the included angle between two MVNSs. Then, a projection measurement of MVNSs is proposed on the basis of the cosine measurement. A normalized projection measurement 
of MVNSs is also presented to cover the defect of the proposed projection measurement.

Definition 7 Let $A=\left\{x_{t},\left(\tilde{t}_{A}\left(x_{t}\right), \tilde{i}_{A}\left(x_{t}\right), \tilde{f}_{A}\left(x_{t}\right)\right)\right\}$ and $B=$ $\left\{x_{t},\left(\tilde{t}_{B}\left(x_{t}\right), \tilde{i}_{B}\left(x_{t}\right), \tilde{f}_{B}\left(x_{t}\right)\right)\right\}$ be two MVNSs. The inner product between $A$ and $B$ can be defined as:

$$
\begin{aligned}
A \cdot B= & \sum_{j=1}^{n}\left(\left(\frac{1}{l_{A t}} \sum_{k=1}^{l_{A t}} t_{A k}\left(x_{j}\right)\right)\left(\frac{1}{l_{B t}} \sum_{k=1}^{l_{B t}} t_{B k}\left(x_{j}\right)\right)\right. \\
& +\left(\frac{1}{l_{A i}} \sum_{g=1}^{l_{A i}}\left(1-i_{A g}\left(x_{j}\right)\right)\right)\left(\frac{1}{l_{B i}} \sum_{g=1}^{l_{B i}}\left(1-i_{B g}\left(x_{j}\right)\right)\right) \\
& \left.+\left(\frac{1}{l_{A f}} \sum_{r=1}^{l_{A f}}\left(1-f_{A r}\left(x_{j}\right)\right)\right)\left(\frac{1}{l_{B f}} \sum_{r=1}^{l_{B f}}\left(1-f_{B r}\left(x_{j}\right)\right)\right)\right),
\end{aligned}
$$

where $l_{A t}, l_{A i}, l_{A f}$ are the numbers of elements in $\tilde{t}_{A}\left(x_{t}\right)$, $\tilde{i}_{A}\left(x_{t}\right)$ and $\tilde{f}_{A}\left(x_{t}\right)$ and $l_{B t}, l_{B i}, l_{B f}$ are the numbers of elements in $\tilde{t}_{B}\left(x_{t}\right), \tilde{i}_{B}\left(x_{t}\right)$ and $\tilde{f}_{B}\left(x_{t}\right)$. The modules of $A$ and $B$ can be defined as:
Proof Let $A=\left\{x_{t},\left(\tilde{t}_{A}\left(x_{t}\right), \tilde{i}_{A}\left(x_{t}\right), \tilde{f}_{A}\left(x_{t}\right)\right)\right\}$ and $B=\left\{x_{t}\right.$, $\left.\left(\tilde{t}_{B}\left(x_{t}\right), \tilde{i}_{B}\left(x_{t}\right), \tilde{f}_{B}\left(x_{t}\right)\right)\right\}$.

1. By Definition $1, t_{A k}\left(x_{j}\right), i_{A g}\left(x_{j}\right), t_{A r}\left(x_{j}\right) \in[0,1]$ exist for any $k \in\left\{1,2, \ldots, l_{A t}\right\}, \quad g \in\left\{1,2, \ldots, l_{A i}\right\}$ and $r \in$ $\left\{1,2, \ldots, l_{A f}\right\}$ and $t_{B k}\left(x_{j}\right), t_{B \mathrm{~g}}\left(x_{j}\right), t_{B r}\left(x_{j}\right) \in[0,1]$ exist for any $k \in\left\{1,2, \ldots, l_{B t}\right\}, \quad g \in\left\{1,2, \ldots, l_{B i}\right\}$ and $r \in\left\{1,2, \ldots, l_{B f}\right\}$. Therefore, it is true that $A \cdot B \geq 0$, $|A| \geq 0$ and $|B| \geq 0$. Thus, $\operatorname{Cos}(A, B)=\frac{A \cdot B}{|A||B|} \geq 0$. By the Cauchy-Schwarz inequality: $\left(y_{1} z_{1}+y_{2} z_{2}+\cdots+\right.$ $\left.y_{n} z_{n}\right)^{2} \leq\left(y_{1}^{2}+y_{2}^{2}+\cdots+y_{n}^{2}\right)\left(z_{1}^{2}+z_{2}^{2}+\cdots+z_{n}^{2}\right)$, we can obtain that $0 \leq A \cdot B \leq|A||B|$. That is to say, $\operatorname{Cos}(A, B)=\frac{A \cdot B}{|A||B|} \leq 1$. Hence, $0 \leq \operatorname{Cos}(A, B) \leq 1$ holds.

2. When $A=B, \quad \frac{1}{l_{A t}} \sum_{k=1}^{l_{A t}} t_{A k}\left(x_{j}\right)=\frac{1}{l_{B t}} \sum_{k=1}^{l_{B t}} t_{B k}\left(x_{j}\right), \quad \frac{1}{l_{A i}}$ $\sum_{g=1}^{l_{A i}}\left(1-i_{A g}\left(x_{j}\right)\right)=\frac{1}{l_{B i}} \sum_{g=1}^{l_{B i}}\left(1-i_{B g}\left(x_{j}\right)\right)$ and $\frac{1}{l_{A f}}$

$$
\begin{aligned}
& |A|=\sqrt{\sum_{j=1}^{n}\left(\left(\frac{1}{l_{A t}} \sum_{k=1}^{l_{A t}} t_{A k}\left(x_{j}\right)\right)^{2}+\left(\frac{1}{l_{A i}} \sum_{g=1}^{l_{A i}}\left(1-i_{A g}\left(x_{j}\right)\right)\right)^{2}+\left(\frac{1}{l_{A f}} \sum_{r=1}^{l_{A f}}\left(1-f_{A r}\left(x_{j}\right)\right)\right)^{2}\right)}, \\
& |B|=\sqrt{\sum_{j=1}^{n}\left(\left(\frac{1}{l_{B t}} \sum_{k=1}^{l_{B t}} t_{B k}\left(x_{j}\right)\right)^{2}+\left(\frac{1}{l_{B i}} \sum_{g=1}^{l_{B i}}\left(1-i_{B g}\left(x_{j}\right)\right)\right)^{2}+\left(\frac{1}{l_{B f}} \sum_{r=1}^{l_{B f}}\left(1-f_{B r}\left(x_{j}\right)\right)\right)^{2}\right)},
\end{aligned}
$$

and the cosine of the included angle between two MVNSs $A$ and $B$ can be defined as:

$$
\begin{aligned}
& \sum_{r=1}^{l_{A f}}\left(1-f_{A r}\left(x_{j}\right)\right)=\frac{1}{l_{B f}} \sum_{r=1}^{l_{B f}}\left(1-f_{B r}\left(x_{j}\right)\right) . \\
& A \cdot B=|A|^{2}=|A||B| \cdot \operatorname{Cos}(A, B)=\frac{A \cdot B}{|A||B|}=1 .
\end{aligned}
$$

Thus,

$$
\begin{aligned}
& \operatorname{Cos}(A, B)=\frac{A \cdot B}{|A||B|} \\
& =\frac{\sum_{j=1}^{n}\left(\left(\frac{1}{l_{A t}} \sum_{k=1}^{l_{A t}} t_{A k}\left(x_{j}\right)\right)\left(\frac{1}{l_{B t}} \sum_{k=1}^{l_{B t}} t_{B k}\left(x_{j}\right)\right)+\left(\frac{1}{l_{A i}} \sum_{g=1}^{l_{A i}}\left(1-i_{A g}\left(x_{j}\right)\right)\right)\left(\frac{1}{l_{B i}} \sum_{g=1}^{l_{B i}}\left(1-i_{B g}\left(x_{j}\right)\right)\right)+\left(\frac{1}{l_{A f}} \sum_{r=1}^{l_{A f}}\left(1-f_{A r}\left(x_{j}\right)\right)\right)\left(\frac{1}{l_{B f}} \sum_{r=1}^{l_{B f}}\left(1-f_{B r}\left(x_{j}\right)\right)\right)\right)}{\sqrt{\sum_{j=1}^{n}\left(\left(\frac{1}{l_{A t}} \sum_{k=1}^{l_{A t}} t_{A k}\left(x_{j}\right)\right)^{2}+\left(\frac{1}{l_{A i}} \sum_{g=1}^{l_{A i}}\left(1-i_{A g}\left(x_{j}\right)\right)\right)^{2}+\left(\frac{1}{l_{A f}} \sum_{r=1}^{l_{A f}}\left(1-f_{A r}\left(x_{j}\right)\right)\right)^{2}\right) \times \sqrt{\sum_{j=1}^{n}\left(\left(\frac{1}{l_{B t}} \sum_{k=1}^{l_{B t}} t_{B k}\left(x_{j}\right)\right)^{2}+\left(\frac{1}{l_{B i}} \sum_{g=1}^{l_{B i}}\left(1-i_{B g}\left(x_{j}\right)\right)\right)^{2}+\left(\frac{1}{l_{B f}} \sum_{r=1}^{l_{B f}}\left(1-f_{B r}\left(x_{j}\right)\right)\right)^{2}\right)}}} .
\end{aligned}
$$

Theorem 1 The cosine of the included angle between two MVNSs A and B satisfies the following properties:

1. $0 \leq \operatorname{Cos}(A, B) \leq 1$;

2. If $A=B$, then $\operatorname{Cos}(A, B)=1$;

3. $\operatorname{Cos}(A, B)=\operatorname{Cos}(B, A)$.
3. By Eq. (4), $\operatorname{Cos}(A, B)=\frac{A \cdot B}{|A||B|}$ and $\operatorname{Cos}(B, A)=\frac{B \cdot A}{|A||B|}$. Since $A \cdot B=B \cdot A, \operatorname{Cos}(A, B)=\operatorname{Cos}(B, A)$.

Therefore, Theorem 1 holds.

The projection measurement of MVNSs is defined based on the proposed cosine measurement as follows. 
Definition 8 Let $A=\left\{x_{t},\left(\tilde{t}_{A}\left(x_{t}\right), \tilde{i}_{A}\left(x_{t}\right), \tilde{f}_{A}\left(x_{t}\right)\right)\right\}$ and $B=$ $\left\{x_{t},\left(\tilde{t}_{B}\left(x_{t}\right), \tilde{i}_{B}\left(x_{t}\right), \tilde{f}_{B}\left(x_{t}\right)\right)\right\}$ be two MVNSs. Then the projection of $A$ on $B$ can be defined as:
Therefore, it is true that $\operatorname{Proj}_{C}(A)=\frac{A \cdot C}{|C|} \leq \frac{B \cdot C}{|C|}=$ $\operatorname{Proj}_{C}(B)$.

3. By Theorem $1, \operatorname{Cos}(A, B)=\operatorname{Cos}(B, A)=1$ when

$$
\begin{aligned}
& \operatorname{Proj}_{B}(A)=|A| \operatorname{Cos}(A, B)
\end{aligned}
$$

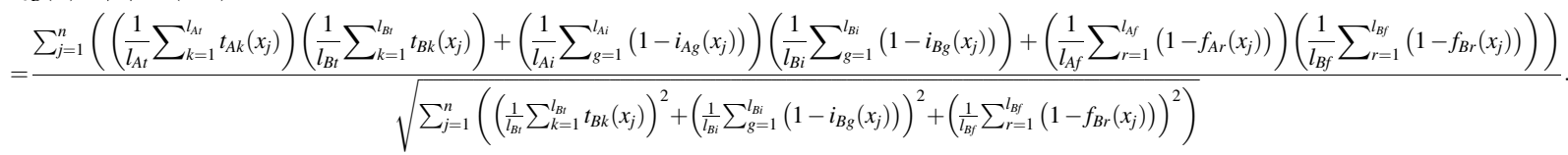

It should be noted that $\operatorname{Proj}_{B}(A) \neq \operatorname{Proj}_{A}(B)$.

Example 4 Let $B=\{x,\{0.3,0.4,0.5\},\{0.2,0.3\},\{0.1\}\}$ and $C=\{x,\{0.4,0.5,0.6\},\{0.2,0.3\},\{0.2\}\} \quad$ be two MVNSs. By Eq. (5), $\operatorname{Proj}_{C}(B)=\frac{0.4 \times 0.5+0.75 \times 0.75+0.9 \times 0.8}{\sqrt{0.5^{2}+0.75^{2}+0.8^{2}}}=$ $\frac{1.4825}{\sqrt{1.4525}}=1.230 \quad$ and $\operatorname{Proj}_{B}(C)=\frac{0.4 \times 0.5+0.75 \times 0.75+0.9 \times 0.8}{\sqrt{0.4^{2}+0.75^{2}+0.9^{2}}}$ $=\frac{1.4825}{\sqrt{1.5325}}=1.198$. It is obvious that $\operatorname{Proj}_{C}(B) \neq \operatorname{Proj}_{B}(C)$.

Theorem 2 Let $A, B$ and $C$ be three MVNSs. Then, the projection measurement of MVNSs satisfies the following properties:

1. $0 \leq \operatorname{Proj}_{B}(A) \leq|A| \leq \sqrt{3 n}$;

2. If $A \subseteq B$, then $\operatorname{Proj}_{C}(A) \leq \operatorname{Proj}_{C}(B)$; and

3. If $A=B$, then $\operatorname{Proj}_{A}(B)=\operatorname{Proj}_{B}(A)=|A|=|B|$.

Proof Let $A=\left\{x_{t},\left(\tilde{t}_{A}\left(x_{t}\right), \tilde{i}_{A}\left(x_{t}\right), \tilde{f}_{A}\left(x_{t}\right)\right)\right\}, \quad B=\left\{x_{t},\left(\tilde{t}_{B}\right.\right.$ $\left.\left.\left(x_{t}\right), \tilde{i}_{B}\left(x_{t}\right), \tilde{f}_{B}\left(x_{t}\right)\right)\right\}$ and $C=\left\{x_{t},\left(\tilde{t}_{C}\left(x_{t}\right), \tilde{i}_{C}\left(x_{t}\right), \tilde{f}_{C}\left(x_{t}\right)\right)\right\}$.

1. By Theorem $1, \operatorname{Cos}(A, B) \in[0,1]$. Since $0 \leq|A|$ $\leq \sqrt{3 n}, \operatorname{Proj}_{B}(A)=|A| \operatorname{Cos}(A, B) \in[0,|A|]$. Therefore, $0 \leq \operatorname{Proj}_{B}(A) \leq|A| \leq \sqrt{3 n}$.

2. When $A \subseteq B, \quad \frac{1}{l_{A t}} \sum_{k=1}^{l_{A t}} t_{A k}\left(x_{j}\right) \leq \frac{1}{l_{B t}} \sum_{k=1}^{l_{B t}} t_{B k}\left(x_{j}\right), \quad \frac{1}{l_{A i}}$ $\sum_{g=1}^{l_{A i}}\left(1-i_{A g}\left(x_{j}\right)\right) \leq \frac{1}{l_{B i}} \sum_{g=1}^{l_{B i}}\left(1-i_{B g}\left(x_{j}\right)\right)$ and $\frac{1}{l_{A f}}$ $\sum_{r=1}^{l_{A f}}\left(1-f_{A r}\left(x_{j}\right)\right) \leq \frac{1}{l_{B f}} \sum_{r=1}^{l_{B f}}\left(1-f_{B r}\left(x_{j}\right)\right)$. Hence,
$A=B$. Moreover, $|A|=|B|$. Thus, $\operatorname{Proj}_{A}(B)=|B|$ Cos $(B, A)=|A| \operatorname{Cos}(A, B)=\operatorname{Proj}_{B}(A)=|A|=|B|$.

Therefore, Theorem 2 is true.

The projection measurement is proposed to reflect the degree that one object is close to another [60]. Generally speaking, the larger $\operatorname{Proj}_{B}(A)$ is, the closer $A$ is to $B$. Nevertheless, the situation is opposite sometimes.

Example 5 Let $A=\{x,\{0.5,0.6,0.7\},\{0.2,0.3\},\{0.3\}\}$ and $B=\{x,\{0.4,0.5,0.6\},\{0.2,0.3\},\{0.2\}\} \quad$ be two MVNSs. By Eq. (5), $\operatorname{Proj}_{B}(A)=\frac{0.6 \times 0.5+0.75 \times 0.75+0.7 \times 0.8}{\sqrt{0.5^{2}+0.75^{2}+0.8^{2}}}=$ $\frac{1.4225}{\sqrt{1.4525}}=1.180$ and $\operatorname{Proj}_{B}(B)=\frac{0.5 \times 0.5+0.75 \times 0.75+0.8 \times 0.8}{\sqrt{0.5^{2}+0.75^{2}+0.8^{2}}}=$ $\frac{1.4525}{\sqrt{1.4525}}=1.205$. It is obvious that $\operatorname{Proj}_{B}(A)>\operatorname{Proj}_{B}(B)$. According to the projection value, $A$ is closer to $B$ than $B$ itself, which does not conform to our intuition.

To cover this deficiency, the normalized projection measurement of MVNSs is presented motivated by Ref. [60] as follows.

Definition 9 Let $B=\left\{x_{t},\left(\tilde{t}_{B}\left(x_{t}\right), \tilde{i}_{B}\left(x_{t}\right), \tilde{f}_{B}\left(x_{t}\right)\right)\right\}$ and $C=\left\{x_{t},\left(\tilde{t}_{C}\left(x_{t}\right), \tilde{i}_{C}\left(x_{t}\right), \tilde{f}_{C}\left(x_{t}\right)\right)\right\}$ be two MVNSs. Then, the normalized projection of $B$ on $C$ can be defined as:

$$
\begin{aligned}
A \cdot C= & \sum_{j=1}^{n}\left(\left(\frac{1}{l_{A t}} \sum_{k=1}^{l_{A t}} t_{A k}\left(x_{j}\right)\right)\left(\frac{1}{l_{C t}} \sum_{k=1}^{l_{C t}} t_{C k}\left(x_{j}\right)\right)+\left(\frac{1}{l_{A i}} \sum_{g=1}^{l_{A i}}\left(1-i_{A g}\left(x_{j}\right)\right)\right)\left(\frac{1}{l_{C i}} \sum_{g=1}^{l_{C i}}\left(1-i_{C g}\left(x_{j}\right)\right)\right)\right. \\
& +\left(\frac{1}{l_{A f}} \sum_{r=1}^{l_{A f}}\left(1-f_{A r}\left(x_{j}\right)\right)\right)\left(\frac{1}{l_{C f}} \sum_{r=1}^{l_{C f}}\left(1-f_{C r}\left(x_{j}\right)\right)\right) \leq \sum_{j=1}^{n}\left(\left(\frac{1}{l_{B t}} \sum_{k=1}^{l_{B t}} t_{B k}\left(x_{j}\right)\right)\left(\frac{1}{l_{C t}} \sum_{k=1}^{l_{C t}} t_{C k}\left(x_{j}\right)\right)\right. \\
& \left.+\left(\frac{1}{l_{B i}} \sum_{g=1}^{l_{B i}}\left(1-i_{B g}\left(x_{j}\right)\right)\right)\left(\frac{1}{l_{C i}} \sum_{g=1}^{l_{C i}}\left(1-i_{C g}\left(x_{j}\right)\right)\right)+\left(\frac{1}{l_{B f}} \sum_{r=1}^{l_{B f}}\left(1-f_{B r}\left(x_{j}\right)\right)\right)\left(\frac{1}{l_{C f}} \sum_{r=1}^{l_{C f}}\left(1-f_{C r}\left(x_{j}\right)\right)\right)\right) \\
= & B \cdot C .
\end{aligned}
$$




$$
\begin{aligned}
\operatorname{NProj}_{C}(B) & =\frac{\operatorname{Proj}_{C}(B) /|C|}{\operatorname{Proj}_{C}(B) /|C|+\left|1-\operatorname{Proj}_{C}(B) /\right| C||} \\
& =\frac{\operatorname{Proj}_{C}(B)}{\operatorname{Proj}_{C}(B)+|| C\left|-\operatorname{Proj}_{C}(B)\right|} .
\end{aligned}
$$

Theorem 3 Let $A, B$ and $C$ be three MVNSs. Then,

1. $0 \leq \operatorname{NProj}_{B}(A) \leq 1$;

2. If $A=B$, then $\operatorname{NProj}_{B}(A)=1$; and

3. If $A \subseteq B \subseteq C$, then $\operatorname{NProj}_{C}(A) \leq \operatorname{NProj}_{C}(B)$.

Proof Let $A=\left\{x_{t},\left(\tilde{t}_{A}\left(x_{t}\right), \tilde{i}_{A}\left(x_{t}\right), \tilde{f}_{A}\left(x_{t}\right)\right)\right\}, \quad B=\left\{x_{t},\left(\tilde{t}_{B}\right.\right.$ $\left.\left.\left(x_{t}\right), \tilde{i}_{B}\left(x_{t}\right), \tilde{f}_{B}\left(x_{t}\right)\right)\right\}$ and $C=\left\{x_{t},\left(\tilde{t}_{C}\left(x_{t}\right), \tilde{i}_{C}\left(x_{t}\right), \tilde{f}_{C}\left(x_{t}\right)\right)\right\}$.

1. By Theorem $2, \operatorname{Proj}_{B}(A) \geq 0$. Therefore, $\operatorname{NProj}_{B}(A)=$ $\frac{\operatorname{Proj}_{B}(A)}{\operatorname{Proj}_{B}(A)+|B|-\operatorname{Proj}_{B}(A) \mid} \geq 0$. Furthermore, $0 \leq \operatorname{Proj}_{B}(A)$ $\leq \operatorname{Proj}_{B}(A)+|| B\left|-\operatorname{Proj}_{B}(A)\right|$. It is true that $\operatorname{NProj}_{B}(A)=\frac{\operatorname{Proj}_{B}(A)}{\operatorname{Proj}_{B}(A)+|| B\left|-\operatorname{Proj}_{B}(A)\right|} \leq 1 . \quad$ Hence, $0 \leq \operatorname{NProj}_{B}(A) \leq 1$ holds.

2. By Theorem 2, $\operatorname{Proj}_{A}(B)=\operatorname{Proj}_{B}(A)=|A|=|B|$ when $A=B$. Thus, $\quad \operatorname{NProj}_{B}(A)=\frac{\operatorname{Proj}_{B}(A)}{\operatorname{Proj}_{B}(A)+|B|-\operatorname{Proj}_{B}(A) \mid}=$ $\frac{|B|}{|B|+0}=1$.

3. When $A \subseteq B \subseteq C, \frac{1}{l_{A t}} \sum_{k=1}^{l_{A t}} t_{A k}\left(x_{j}\right) \leq \frac{1}{l_{B t}} \sum_{k=1}^{l_{B t}} t_{B k}\left(x_{j}\right)$ $\leq \frac{1}{l_{C t}} \sum_{k=1}^{l_{C t}} t_{C k}\left(x_{j}\right), \quad \frac{1}{l_{A i}} \sum_{g=1}^{l_{A i}}\left(1-i_{A g}\left(x_{j}\right)\right) \leq \frac{1}{l_{B i}} \sum_{g=1}^{l_{B i}}$ $\left(1-i_{B g}\left(x_{j}\right)\right) \leq \frac{1}{l_{C i}} \sum_{g=1}^{l_{C i}}\left(1-i_{C g}\left(x_{j}\right)\right) \quad$ and $\quad \frac{1}{l_{A f}} \sum_{r=1}^{l_{A f}}$ $\left(1-f_{A r}\left(x_{j}\right)\right) \leq \frac{1}{l_{B f}} \sum_{r=1}^{l_{B f}}\left(1-f_{B r}\left(x_{j}\right)\right) \leq \frac{1}{l_{C f}} \sum_{r=1}^{l_{C f}}(1-$ $\left.f_{C r}\left(x_{j}\right)\right)$. Therefore, $|A| \leq|B| \leq|C|$. By Theorem 2, $\operatorname{NProj}_{C}(A) \leq|A| \leq|C|$ and $\operatorname{NProj}_{C}(B) \leq|B| \leq|C|$. It can be obtained that $\operatorname{NProj}_{C}(A)=$ $\frac{\operatorname{Proj}_{C}(A)}{\operatorname{Proj}_{C}(A)+|C|-\operatorname{Proj}_{C}(A) \mid}=\frac{\operatorname{Proj}_{C}(A)}{|C|}$ and $\operatorname{NProj}_{C}(B)=$ $\frac{\operatorname{Proj}_{C}(B)}{\operatorname{Proj}_{C}(B)+|C|-\operatorname{Proj}_{C}(B) \mid}=\frac{\operatorname{Proj}_{C}(B)}{|C|}$. By Theorem 2, $\operatorname{Proj}_{C}(A) \leq \operatorname{Proj}_{C}(B)$. Hence, $\operatorname{NProj}_{C}(A) \leq \operatorname{NProj}_{C}(B)$.

Example 6 Let $A=\{x,\{0.1,0.2,0.3\},\{0.4,0.5\},\{0.6\}\}$, $B=\{x,\{0.3,0.5,0.7\},\{0.3,0.4\},\{0.4\}\} \quad$ and $C=\{x$, $\{0.5,0.6,0.7\},\{0.3,0.4\},\{0.3\}\}$ be three MVNSs. By Eq. (5), $\operatorname{Proj}_{B}(A)=\frac{0.2 \times 0.5+0.55 \times 0.65+0.4 \times 0.6}{\sqrt{0.5^{2}+0.65^{2}+0.6^{2}}}=\frac{0.6975}{\sqrt{1.0325}}=$ 0.686, $|B|=\sqrt{1.0325}, \operatorname{Proj}_{C}(A)=\frac{0.2 \times 0.6+0.55 \times 0.65+0.4 \times 0.7}{\sqrt{0.6^{2}+0.65^{2}+0.7^{2}}}$ $=\frac{0.7575}{\sqrt{1.2725}}=0.672$, and $|C|=\sqrt{1.2725}$. By Eq. (6), $\operatorname{NProj}_{B}(A)=\frac{0.686}{0.686+|\sqrt{1.0325}-0.686|}=0.675$ and $\operatorname{NProj}_{C}(A)=$ $0.672 /(0.672+|\sqrt{1.2725-0.672}|)=0.596$. Therefore, $\operatorname{NProj}_{C}(A)<\operatorname{NProj}_{B}(A)$, that is, $A$ is closer to $B$ than to $C$.

\subsection{The projection-based difference measurement of MVNSs}

In this subsection, a difference measurement of MVNSs is defined based on the projection and normalized projection measurements in Sect. 3.2 to denote the difference between two MVNSs.

Definition 10 Let $B=\left\{x_{t},\left(\tilde{t}_{B}\left(x_{t}\right), \tilde{i}_{B}\left(x_{t}\right), \tilde{f}_{B}\left(x_{t}\right)\right)\right\}$ and $C=\left\{x_{t},\left(\tilde{t}_{C}\left(x_{t}\right), \tilde{i}_{C}\left(x_{t}\right), \tilde{f}_{C}\left(x_{t}\right)\right)\right\}$ be two MVNSs. Then, the projection-based difference between $B$ and $C$ can be defined as:

$\operatorname{Diff}(B, C)=\operatorname{NProj}_{I}(B)-\operatorname{NProj}_{I}(C)$.

where $I$ is any MVNS satisfying $B \subseteq I$ and $C \subseteq I$.

Theorem 4 Let $B=\left\{x_{t},\left(\tilde{t}_{B}\left(x_{t}\right), \tilde{i}_{B}\left(x_{t}\right), \tilde{f}_{B}\left(x_{t}\right)\right)\right\}$ and $C=$ $\left\{x_{t},\left(\tilde{t}_{C}\left(x_{t}\right), \tilde{i}_{C}\left(x_{t}\right), \tilde{f}_{C}\left(x_{t}\right)\right)\right\}$ be two MVNSs. The projectionbased difference between $B$ and $C$ can be defined as:

$\operatorname{Diff}(B, C)=\frac{\operatorname{Proj}_{I}(B)-\operatorname{Proj}_{I}(C)}{|I|}$.

Proof By Definition 10, $B \subseteq I$ and $C \subseteq I$. Therefore, $|B| \leq|I|$ and $|C| \leq|I|$. By Theorem 2, it is true that $\operatorname{Proj}_{I}(B) \leq|B| \leq|I|$ and $\operatorname{Proj}_{I}(C) \leq|C| \leq|I|$. By Eq. (6), $\operatorname{NProj}_{I}(B)=\frac{\operatorname{Proj}_{I}(B)}{\operatorname{Proj}_{I}(B)+|I|-\operatorname{Proj}_{I}(B) \mid}=\frac{\operatorname{Proj}_{I}(B)}{|I|}$ and $\operatorname{NProj}_{I}(C)$ $=\frac{\operatorname{Proj}_{I}(C)}{\operatorname{Proj}_{I}(C)+|| I\left|-\operatorname{Proj}_{I}(C)\right|}=\frac{\operatorname{Proj}_{I}(C)}{|I|}$. Thus, by Eq. (7), Diff $(B, C)=\operatorname{NProj}_{I}(B)-\operatorname{NProj}_{I}(C)=\frac{\operatorname{Proj}_{I}(B)-\operatorname{Proj}_{I}(C)}{|I|}$.

Theorem 5 The projection-based difference measurement between two MVNSs $B$ and $C$ satisfies the following properties:

1. $-1 \leq \operatorname{Diff}(B, C) \leq 1$;

2. If $B=C$, then $\operatorname{Diff}(B, C)=0$;

3. If $B \subseteq C$, then $\operatorname{Diff}(B, C) \leq 0$;

4. If $C \subseteq B$, then $\operatorname{Diff}(B, C) \geq 0$;

5. $\operatorname{Diff}(B, C)+\operatorname{Diff}(C, B)=0$;

6. If $\operatorname{Diff}(B, C)+\operatorname{Diff}(C, B)=0$, then $\operatorname{Diff}(B, C)=$ $\operatorname{Diff}(C, B)=0$; and

7. If $\operatorname{Diff}(B, C)>0$ and $\operatorname{Diff}(C, D)>0$, then $\operatorname{Diff}(B, D)$ $>0$.

\section{Proof}

1. By Theorem 3, $0 \leq \operatorname{NProj}_{I}(B) \leq 1$ and $0 \leq \operatorname{NProj}_{I}$ $(C) \leq 1$. Thus, $\quad \operatorname{Diff}(B, C)=\operatorname{Proj}_{I}(B)-\operatorname{Proj}_{I}(C) \in$ $[-1,1]$.

2. When $B=C$, we have that $\operatorname{NProj}_{I}(B)=\operatorname{NProj}_{I}(C)$. Hence, $\operatorname{Diff}(B, C)=\operatorname{NProj}_{I}(B)-\operatorname{NProj}_{I}(C)=0$. 
3. By Theorem 3, $\operatorname{NProj}_{I}(B) \leq \operatorname{NProj}_{I}(C)$ when $B \subseteq C$. Therefore, $\operatorname{Diff}(B, C)=\operatorname{NProj}_{I}(B)-\operatorname{NProj}_{I}(C) \leq 0$.

4. By Theorem 3, $\operatorname{NProj}_{I}(B) \geq \operatorname{NProj}_{I}(C)$ when $C \subseteq B$. Therefore, $\operatorname{Diff}(B, C)=\operatorname{NProj}_{I}(B)-\operatorname{NProj}_{I}(C) \geq 0$.

5. By Eq. (7), $\operatorname{Diff}(B, C)=\operatorname{NProj}_{I}(B)-\operatorname{NProj}_{I}(C)$ and $\operatorname{Diff}(C, B)=\operatorname{NProj}_{I}(C)-\operatorname{NProj}_{I}(B)$. Therefore, Diff $(B, C)+\operatorname{Diff}(C, B)=\left(\operatorname{NProj}_{I}(B)-\operatorname{NProj}_{I}(C)\right)+$ $\left(\operatorname{NProj}_{I}(C)-\operatorname{NProj}_{I}(B)\right)=0$.

6. By (3), $\operatorname{Diff}(B, C)+\operatorname{Diff}(C, B)=0$. When $\operatorname{Diff}(B, C)$ $=\operatorname{Diff}(C, B), \operatorname{Diff}(B, C)=\operatorname{Diff}(C, B)=0$.

7. When $\operatorname{Diff}(B, C)>0, \quad \operatorname{NProj}_{I}(B)-\operatorname{NProj}_{I}(C)>0$, that is $\operatorname{NProj}_{I}(B)>\operatorname{NProj}_{I}(C)$. When $\operatorname{Diff}(C, D)>$ $0, \operatorname{NProj}_{I}(C)-\operatorname{NProj}_{I}(D)>0$, that is $\operatorname{NProj}_{I}(C)>$ $\operatorname{NProj}_{I}(D)$. Thus, $\operatorname{NProj}_{I}(B)>\operatorname{NProj}_{I}(C)>\operatorname{NProj}_{I}$ $(D)$, and $\operatorname{Diff}(B, D)=\operatorname{NProj}_{I}(B)-\operatorname{NProj}_{I}(D)>0$.

Therefore, Theorem 4 holds.

Example 7 Let $A=\{x,\{0.1,0.2,0.3\},\{0.4,0.5\},\{0.6\}\}$ and $B=\{x,\{0.3,0.5,0.7\},\{0.3,0.4\},\{0.4\}\}$ be two MVNSs. Let $I=\{x,\{0.7\},\{0.3\},\{0.4\}\}$. It is obvious that $A \subseteq I \quad$ and $\quad B \subseteq I . \quad$ By Eq. (5), $\quad \operatorname{Proj}_{I}(A)=$ $\frac{0.2 \times 0.7+0.55 \times 0.7+0.4 \times 0.6}{\sqrt{0.7^{2}+0.7^{2}+0.6^{2}}}=\frac{0.765}{\sqrt{1.34}}=0.661, \quad \operatorname{Proj}_{I}(B)=$ $\frac{0.5 \times 0.7+0.65 \times 0.7+0.6 \times 0.6}{\sqrt{0.7^{2}+0.7^{2}+0.6^{2}}}=\frac{1.165}{\sqrt{1.34}}=1.006$, and $|I|=\sqrt{1.34}$. By Eq. (6), $\quad \operatorname{NProj}_{I}(A)=\frac{0.661}{\sqrt{1.34}}=0.571$ and $\operatorname{NProj}_{I}(B)=$ $\frac{1.006}{\sqrt{1.34}}=0.869$. Consequently, by Eq. (7), $\operatorname{Diff}(A, B)=$ $\operatorname{NProj}_{I}(A)-\mathrm{NProj}_{I}(B)=0.571-0.869=-0.298$.

\subsection{A projection-based TODIM method for MCDM problems}

In this subsection, we construct a projection-based TODIM method for MCDM problems with MVNSs to cover the shortage demonstrated in Sect. 2.2. The projection-based TODIM method introduces the projectionbased difference measurement to depict the distinction between objects.

Assume there are $m$ alternatives $A=\left\{A_{1}, A_{2}, \ldots A_{m}\right\}$ and $n$ criteria $C=\left\{C_{1}, C_{2}, \ldots C_{n}\right\}$. To find the most desirable alternative, $m$ alternatives are evaluated by decision-makers concerning $n$ criteria. Considering the fuzziness, the evaluations are transformed into MVNNs, and the transformed decision matrix can be denoted as:

$U=\left(\begin{array}{cccc}U_{11} & U_{12} & \cdots & U_{1 n} \\ U_{21} & U_{22} & \cdots & U_{2 n} \\ \vdots & \vdots & \ddots & \vdots \\ U_{m 1} & U_{m 2} & \cdots & U_{m n}\end{array}\right)$, where $\quad U_{r j}=\left\{\tilde{t}_{r j}, \tilde{i}_{r j}, \tilde{f}_{r j}\right\} \quad\left(\tilde{t}_{r j}=\left\{t_{r j}^{1}, t_{r j}^{2}, \ldots, t_{r j}^{l_{r j}}\right\}, \tilde{i}_{r j}=\right.$ $\left.\left\{i_{r j}^{1}, i_{r j}^{2}, \ldots, i_{r j}^{l_{r j}}\right\}, \tilde{f}_{r j}=\left\{f_{r j}^{1}, f_{r j}^{2}, \ldots, f_{r j}^{l_{F_{r j}}}\right\}\right)$ is the evaluation in the form of MVNNs for the alternative $A_{r}(r=1,2$, $\ldots, m)$ under the criterion $C_{j}(j=1,2, \ldots, n)$. Moreover, the weight vector of criteria is $w=\left(w_{1}, w_{2}, \ldots, w_{n}\right)^{T}$, where $w_{j} \geq 0(j=1,2, \ldots, n)$ and $\sum_{j=1}^{n} w_{j}=1$.

The procedure of the projection-based TODIM method is introduced in the rest of this subsection:

Step 1 Normalize the decision matrix.

Since cost and benefit criteria may exist in an MCDM problem simultaneously, the decision matrix needs to be normalized. When $C_{j}$ is a cost criterion, $U_{r j}$ should be normalized by utilizing the complementary set of MVNNs in Definition 6. When $C_{j}$ is a benefit criterion, $U_{r j}$ is unnecessary to be normalized. The formula of normalization is defined as:

$$
\begin{aligned}
N_{r j} & =\left\{T_{r j}, I_{r j}, F_{r j}\right\} \\
& = \begin{cases}U_{r j} & \text { if } C_{j} \text { is a benefit criterion } \\
\operatorname{neg}\left(U_{r j}\right) & \text { if } C_{j} \text { is a cost criterion. }\end{cases}
\end{aligned}
$$

where $T_{r j}=\left\{T_{r j}^{1}, T_{r j}^{2}, \ldots, T_{r j}^{l_{r j}}\right\}, I_{r j}=\left\{I_{r j}^{1}, I_{r j}^{2}, \ldots, I_{r j}^{l_{I j}}\right\}$ and $F_{r j}=\left\{F_{r j}^{1}, F_{r j}^{2}, \ldots, F_{r j}^{l_{F_{r j}}}\right\} . l_{T_{r j}}, l_{I_{r j}}$ and $l_{F_{r j}}$ are the number of elements in $T_{r j}, I_{r j}$ and $F_{r j}$, respectively.

Step 2 Calculate the ideal alternative.

Here, the ideal alternative is defined as:

$$
I=\left\{\left\{\max _{r, j}\left(T_{r j}^{l_{T_{r j}}}\right)\right\},\left\{\max _{r, j}\left(I_{r j}^{l_{I_{j}}}\right)\right\},\left\{\max _{r, j}\left(F_{r j}^{l_{F_{r j}}}\right)\right\}\right\} .
$$

It is evident that $N_{r j} \subseteq I$ holds for any $r \in\{1,2, \ldots, n\}$ and $j \in\{1,2, \ldots, m\}$.

Step 3 Calculate score values.

The score value $s\left(N_{r j}\right)(r=1,2, \ldots, m ; j=1,2, \ldots, n)$ of $N_{r j}$ can be calculated by the definition of the score function in Definition 4.

Step 4 Calculate accuracy values.

The accuracy value $h\left(N_{r j}\right)(r=1,2, \ldots, m ; j=$ $1,2, \ldots, n)$ of $N_{r j}$ can be calculated by the accuracy function in Definition 5.

Step 5 Obtain projection values.

The projection value $\operatorname{Proj}_{I}^{j}(r)(r=1,2, \ldots, m ; j=$ $1,2, \ldots, n)$ of alternative $A_{r}$ on $I$ concerning the criterion $C_{j}$ can be obtained by Eq. (5). 
Step 6 Obtain the projection-based difference matrices.

The projection-based difference $\operatorname{Diff}_{g}^{j}(r)(r=1,2, \ldots$, $m ; \quad g=1,2, \ldots, m ; \quad j=1,2, \ldots, n)$ between alternatives $A_{r}$ and $A_{g}$ concerning the criterion $C_{j}$ can be obtained by Eq. (8), and the projection-based difference matrices can be obtained.

Step 7 Obtain partial dominance matrices.

The partial dominance matrix $\Phi^{j}$ under the criterion $C_{j}$ is composed of partial dominance degrees $\Phi_{r g}^{j}(r=1,2$, $\ldots, m ; g=1,2, \ldots, m ; j=1,2, \ldots, n)$ of the alternative $A_{r}$ over the alternative $A_{g}$ concerning the criterion $C_{j}$. The partial dominance degree $\Phi_{r g}^{j}$ can be calculated utilizing the obtained projection-based difference matrices:

$\Phi_{r g}^{j}= \begin{cases}\sqrt{\frac{\omega_{j u}}{\sum_{j=1}^{n} \omega_{j u}}} \cdot \operatorname{Diff}_{g}^{j}(r), & N_{r j} \succ N_{g j} \\ 0, & N_{r j}=N_{g j}, \\ \frac{-1}{t} \sqrt{\frac{\sum_{j=1}^{n} \omega_{j u}}{\omega_{j u}}} \cdot \operatorname{Diff}_{r}^{j}(g), & N_{r j} \prec N_{g j}\end{cases}$

where $\omega_{j u}=\frac{\omega_{j}}{\omega_{u}}$ and $\omega_{u}=\max \left(\omega_{j}\right)(j=1,2, \ldots, n)$. If $N_{r j} \succ N_{g j}$, it can be thought as a gain; if $N_{r j}=N_{g j}$, it is breakeven; if $N_{r j} \prec N_{g j}$, it can be thought as a loss. The parameter $t$ is the decay factor of the loss and $t>0$.

Step 8 Obtain the final dominance matrix $\Phi$.

The final dominance matrix $\Phi$ is composed of dominance degrees. The dominance degree $\Phi_{r g}(r=1,2$, $\ldots, m ; g=1,2, \ldots, m ; j=1,2, \ldots, n)$ denotes the degree that the alternative $A_{i}$ is better than the alternative $A_{r}$ and can be obtained by:

$\Phi_{r g}=\sum_{j=1}^{n} \Phi_{r g}^{j}$.

Step 9 Calculate the global values.

The global value $\xi_{r}(r=1,2, \ldots, m)$ of the alternative $A_{r}$ can be obtained by:

$$
\xi_{r}=\frac{\sum_{g=1}^{m} \Phi_{r g}-\min _{1 \leq r \leq m}\left(\sum_{g=1}^{m} \Phi_{r g}\right)}{\max _{1 \leq r \leq m}\left(\sum_{g=1}^{m} \Phi_{r g}\right)-\min _{1 \leq r \leq m}\left(\sum_{g=1}^{m} \Phi_{r g}\right)} .
$$

Step 10 Rank the alternatives.

The ranking order of the alternatives can be obtained according to the global values. The bigger the global value of an individual alternative, the better the alternative will be.

\section{An numerical example}

In this section, a numerical example of the personnel selection problem from Ref. [61] is provided to demonstrate the applicability of the projection-based TODIM method.

Personnel selection is considered as a significant issue for companies because of its influence on the quantity of products and services. Personnel selection is a process of selecting employees whose skills mostly match the position. This process can be thought as an MCDM one: candidates are evaluated by the company under several criteria, including the oral communication skill, the working experience and the general aptitude. Moreover, in view of the fuzziness and hesitancy in the selection process, it would be better to introduce MVNSs to denote evaluations.

Here, let us consider a personnel selection problem that a manufacturing company plans to employ a sales supervisor. After preliminary election from dozens of candidates, four candidates enter the final round interview $A_{r}(r=1,2,3,4)$. The company interviews the four candidates and evaluated these candidates under three criteria: (1) $C_{1}$ is the oral communication skill; (2) $C_{2}$ is the working experience; (3) $C_{3}$ is the general aptitude. The weight vector of criteria is given by the company as $w=(0.35,0.25,0.4)^{T}$. The evaluations of the four candidates under each criterion are transformed into MVNNs, and Table 1 lists the transformed decision-making matrix.

\subsection{The steps of the proposed method}

Step 1 Normalize the decision matrix.

Since all these criteria are benefit ones, it is unnecessary to normalize the decision-making matrix.

Table 1 The transformed decision-making matrix

\begin{tabular}{llll}
\hline & $C_{1}$ & $C_{2}$ & $C_{3}$ \\
\hline$A_{1}$ & $\{\{0.3,0.4,0.5\},\{0.1\},\{0.3,0.4\}\}$ & $\{\{0.5,0.6\},\{0.2,0.3\},\{0.3,0.4\}\}$ & $\{\{0.2,0.3\},\{0.1,0.2\},\{0.5,0.6\}\}$ \\
$A_{2}$ & $\{\{0.6,0.7\},\{0.1,0.2\},\{0.2,0.3\}\}$ & $\{\{0.6,0.7\},\{0.1\},\{0.3\}\}$ & $\{\{0.6,0.7\},\{0.1,0.2\},\{0.1,0.2\}\}$ \\
$A_{3}$ & $\{\{0.5,0.6\},\{0.4\},\{0.2,0.3\}\}$ & $\{\{0.6\},\{0.3\},\{0.4\}\}$ & $\{\{0.5,0.6\},\{0.1\},\{0.3\}\}$ \\
$A_{4}$ & $\{\{0.7,0.8\},\{0.1\},\{0.1,0.2\}\}$ & $\{\{0.6,0.7\},\{0.1\},\{0.2\}\}$ & $\{\{0.3,0.5\},\{0.2\},\{0.1,0.2,0.3\}\}$ \\
\hline
\end{tabular}


Step 2 Calculate the ideal alternative.

By Eq. (10), the ideal alternative $I$ can be calculated as $I=\{\{0.8\},\{0.1\},\{0.1\}\}$.

Step 3 Calculate score values.

By Eq. (1), we can obtain the score value of each alternative concerning each criterion and these score values are presented in Table 2.

Step 4 Calculate accuracy values.

By Eq. (2), we can obtain the accuracy value of each alternative concerning each criterion and these accuracy values are presented in Table 3 .

Step 5 Obtain projection values.

The projection value $\operatorname{Proj}_{I}^{j}(r)(r=1,2,3,4 ; j=1,2,3)$ of alternative $A_{r}$ on $I$ concerning criterion $C_{j}$ can be obtained by Eq. (5), and these projection values are shown in Table 4.

Step 6 Obtain the projection-based difference matrices.

The projection-based difference

$\operatorname{Diff}_{g}^{j}(r)(r=1,2,3,4 ; g=1,2,3,4 ; j=1,2,3,4)$

between alternative $A_{r}$ and $A_{g}$ with respect to criterion $C_{j}$ can be obtained by Eq. (7), and the projection-based difference matrices can be obtained:

Table 2 The score value of each alternative concerning each criterion

Table 3 The accuracy value of each alternative concerning each criterion

Table 4 The projection value of each alternative on I concerning each criterion

$$
\begin{aligned}
& \text { Diff }^{1}=\left(\begin{array}{cccc}
0 & -0.1084 & 0.0265 & -0.2035 \\
0.1084 & 0 & 0.135 & -0.0951 \\
-0.0265 & -0.135 & 0 & -0.2301 \\
0.2035 & 0.0951 & 0.2301 & 0
\end{array}\right), \\
& \operatorname{Diff}^{2}=\left(\begin{array}{cccc}
0 & -0.115 & 0.0221 & -0.155 \\
0.115 & 0 & 0.1372 & -0.04 \\
-0.0221 & -0.1372 & 0 & -0.177 \\
0.1549 & 0.0398 & 0.177 & 0
\end{array}\right), \\
& \text { and Diff }
\end{aligned}
$$

Step 7 Obtain partial dominance matrices.

We can obtain the partial dominance partial dominance degrees $\Phi_{r g}^{j}(r=1,2,3,4 ; g=1,2,3,4 ; j=1,2,3) \quad$ by Eq. (11), and the obtained partial dominance matrices are listed as follows:

$$
\begin{gathered}
\Phi^{1}=\left(\begin{array}{cccc}
0 & -0.1832 & 0.0157 & -0.344 \\
0.0641 & 0 & 0.0798 & -0.1608 \\
-0.045 & -0.2281 & 0 & -0.3889 \\
0.1204 & 0.0563 & 0.1361 & 0
\end{array}\right), \\
\Phi^{2}=\left(\begin{array}{cccc}
0 & -0.2301 & 0.0111 & -0.3097 \\
0.0575 & 0 & 0.0686 & -0.0796 \\
-0.0442 & -0.2743 & 0 & -0.354 \\
0.0774 & 0.0199 & 0.0885 & 0
\end{array}\right), \\
\text { and } \Phi^{3}=\left(\begin{array}{cccc}
0 & -0.4757 & -0.3568 & -0.2729 \\
0.1903 & 0 & 0.0476 & 0.0812 \\
0.1427 & -0.1189 & 0 & 0.0336 \\
0.1091 & -0.2029 & -0.084 & 0
\end{array}\right) .
\end{gathered}
$$

Step 8 Obtain the final dominance matrix $\Phi$.

The dominance degree $\Phi_{r g}(r=1,2,3,4 ; g=1,2,3,4)$ can be obtained by Eq. (12), and the final dominance matrix is:

$$
\Phi=\left(\begin{array}{cccc}
0 & -0.8891 & -0.33 & -0.9266 \\
0.312 & 0 & 0.196 & -0.1593 \\
0.0536 & -0.6214 & 0 & -0.7093 \\
0.307 & -0.1267 & 0.1407 & 0
\end{array}\right)
$$

Step 9 Calculate the global values.

The global value $\xi_{i}(i=1,2,3,4)$ can be obtained by Eq. (13): $\xi_{1}=0, \xi_{2}=1, \xi_{3}=0.3482$ and $\xi_{4}=0.9889$.

Step 10 Rank the alternatives.

Since $\xi_{2}>\xi_{4}>\xi_{3}>\xi_{1}$, the ranking order of the four candidates is $A_{2} \succ A_{4} \succ A_{3} \succ A_{1}$. Thus, the best candidate is $A_{2}$. 


\subsection{The influences of the parameter $t$}

In this subsection, the influence of the parameter $t$ is investigated and discussed in detail.

As narrated in Sect. 3.4, the value of the parameter $t$ in Eq. (11) can influence the partial dominance degrees when there is a loss. That is to say, the value of $t$ can affect the shape of the prospect value function. To show this influence, Fig. 1 depicts the prospect value functions with two different values of $t$, i.e., $t=1$ [62] and $t=2.5$ [51]. In Fig. 1, the horizontal axis represents the projection-based difference between two alternatives concerning the same criterion and the vertical axis represents the corresponding partial dominance degree.

From Fig. 1, the value of $t$ does influence the shape of the prospect value function. The shapes of the prospect value functions with $t=1$ and $t=2.5$ are same in the first quadrant, while the prospect value functions with $t=1$ and $t=2.5$ in the third quadrant have different shapes. Moreover, the shape is deeper when $t=1$ than that when $t=2.5$. The reasons for this phenomenon are explained as follows. From Eq. (11), it is easy to see that the value of $t$ cannot influence the partial dominance degrees when there is a gain, that is, the value of $t$ makes no difference to the shape of the prospect value function in the first quadrant. In addition, From Eq. (11), we know that the greater the value of $t$, the greater the value of the partial dominance

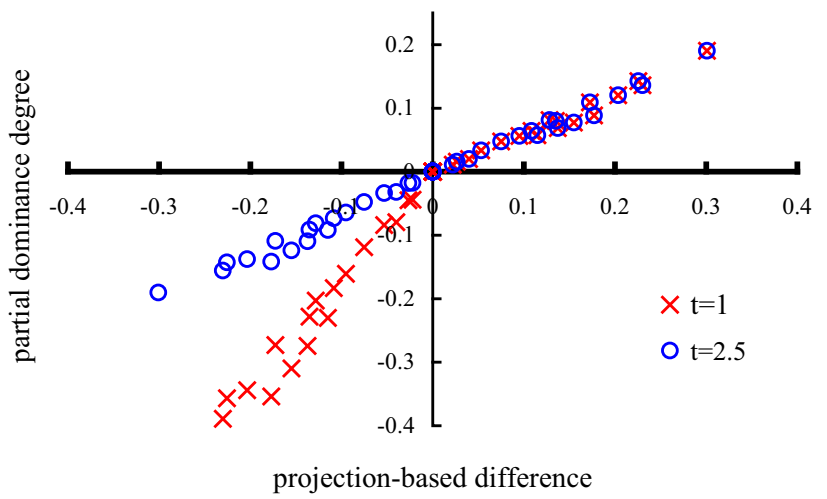

Fig. 1 The prospect value functions with $t=1$ and $t=2.5$ degree will be when there is a loss. Therefore, it is reasonable that the shape of the prospect value function is affected by the value of $t$, and the shape will become shallower with the increase of $t$.

Furthermore, the influence of the parameter $t$ on the ranking order is investigated by comparing the ranking orders obtained with varying values of $t$. As the value of $t$ changes from 0.001 to 50 , the corresponding ranking order of the four candidates can be obtained and compared. Table 5 lists the value of $t$, the corresponding global values, and the ranking order of the candidates.

From Table 5, the ranking order of these four candidates may be distinct with the change of the value of $t$. When $t \leq 2$, a same ranking order is obtained with the change of $t$ and the candidate $A_{2}$ is the best one while the candidate $A_{1}$ is the least desirable. A ranking order, which is different from the order when $t \leq 2$, is obtained when $3 \leq t$. The best candidate becomes $A_{4}$ and $A_{1}$ is still the worst candidate. The reason for these differences is listed as follows. From Eq. (11), we can know that when $t \leq 1$, the losses are amplified and the degree of amplification increases as the value of $t$ decreases. When $t>1$, the losses are attenuated and the degree of attenuation increases as the increase of $t$. In this numerical example, the losses are attenuated when $1<t \leq 2$, and the degree of attenuation is too small to make the ranking order of the four candidates different from the order when $t \leq 1$. What is more, when $3 \leq t$, the degree of attenuation becomes bigger than that when $1<t \leq 2$, and the attenuation of losses makes the candidate $A_{4}$ become better than the candidate $A_{2}$.

In general, the value of $t$ reflects the risk preference of decision-makers and may eventually influence not only the shape of the prospect value function but also the ranking order of the four candidates.

\subsection{Comparative analysis}

In this subsection, we conduct a comparative analysis aiming to certify the feasibility of the projection-based TODIM method. The comparative analysis compares the proposed projection-based TODIM method with three other MCDM methods under multi-valued neutrosophic
Table 5 Ranking orders with different values of $t$

\begin{tabular}{lll}
\hline$t$ & The global value $\xi_{i}$ & Ranking order \\
\hline$t=0.001$ & $\xi_{1}=0, \xi_{2}=1, \xi_{3}=0.3722, \xi_{4}=0.976$ & $A_{2} \succ A_{4} \succ A_{3} \succ A_{1}$ \\
$t=0.1$ & $\xi_{1}=0, \xi_{2}=1, \xi_{3}=0.3692, \xi_{4}=0.9776$ & $A_{2} \succ A_{4} \succ A_{3} \succ A_{1}$ \\
$t=1$ & $\xi_{1}=0, \xi_{2}=1, \xi_{3}=0.3482, \xi_{4}=0.9889$ & $A_{2} \succ A_{4} \succ A_{3} \succ A_{1}$ \\
$t=2$ & $\xi_{1}=0, \xi_{2}=1, \xi_{3}=0.3331, \xi_{4}=0.9971$ & $A_{2} \succ A_{4} \succ A_{3} \succ A_{1}$ \\
$t=3$ & $\xi_{1}=0, \xi_{2}=0.9973, \xi_{3}=0.3218, \xi_{4}=1$ & $A_{4} \succ A_{2} \succ A_{3} \succ A_{1}$ \\
$t=10$ & $\xi_{1}=0, \xi_{2}=0.9817, \xi_{3}=0.2877, \xi_{4}=1$ & $A_{4} \succ A_{2} \succ A_{3} \succ A_{1}$ \\
$t=50$ & $\xi_{1}=0, \xi_{2}=0.9713, \xi_{3}=0.2649, \xi_{4}=1$ & $A_{4} \succ A_{2} \succ A_{3} \succ A_{1}$ \\
\hline
\end{tabular}


Table 6 Ranking orders of the four methods

\begin{tabular}{ll}
\hline Method & Ranking order \\
\hline The first method with MVNWA operator [41] & $A_{4} \succ A_{2} \succ A_{3} \succ A_{1}$ \\
The second method [42] & $A_{2} \succ A_{4} \succ A_{3} \succ A_{1}$ \\
The third method ( $t=1)$ [39] & $A_{2} \succ A_{4} \succ A_{1} \succ A_{3}$ \\
The proposed method $(t=1)$ & $A_{2} \succ A_{4} \succ A_{3} \succ A_{1}$ \\
\hline
\end{tabular}

environments. The first MCDM method is the method proposed by Ye [41]. Ye [41] defined the MVNWA operator and established an MCDM method utilizing the proposed operator. Furthermore, the ranking order of the method in Ref. [41] is obtained by the cosine between the collective value and the ideal element $\{\{1\},\{0\},\{0\}\}$. The second method is the method proposed by Sahin and Liu [42]. Sahin and Liu [42] presented an MCDM method based on the proposed correlation coefficient. Moreover, the correlation coefficient in Ref. [42] adds some elements in MVNSs to make two MVNSs be of same length. The third method is the method proposed by Wang et al. [39]. Wang et al. [39] constructed a TODIM method which is based on the distance measurement. These three methods are used to solve the personnel selection problem in this numerical example, and Table 6 lists the ranking orders of these three methods and the proposed method.

From Table 6 , the best candidate is $A_{4}$ for the first method with the MVNWA operator, while $A_{2}$ is the best one for the rest three methods. The worst candidate is $A_{1}$ for the first two methods and the proposed method, while $A_{3}$ is the worst one for the third method. We give the reasons why the differences exist as follows.

The first method does not consider the risk preference of decision-makers, while the proposed method does. Therefore, it is reasonable that the ranking order of the first method may not be the same as that of the proposed method. In the second method, the selection of the elements added to MVNNs reflects the risk preference of decision-makers to a certain extent. In addition, the comparison method used in the second method differs from that in the proposed method. The ranking orders of these two methods may be different with the change of $t$ though the ranking orders of the second method and the proposed method are same in Table 6 . The third method makes use of the distance measurement, while the proposed method takes advantage of the projection-based difference measurement. The distance measurement cannot take into account the included angle between two MVNNs, while the projection-based difference measurement can. In addition, different comparison methods are used in the third method and the proposed method. Consequently, the third method and the proposed method may have different ranking orders even with the same value of $t$.
Generally speaking, the proposed method can effectively tackle MCDM problems (such as personnel selection) under multi-valued neutrosophic environments. Compared with extant methods, the proposed method takes into account the risk preference and considers both the distance and the included angle between two MVNNs. What is more, the proposed method utilizes an improved comparison method which covers the defect of the extant comparison method. The ranking order of the proposed method is more in line with decision-makers' preferences than those obtained by extant methods.

\section{Conclusion}

MVNSs can better depict fuzzy information in practical problems than FSs, IFSs, NSs and HFSs. Compared with the distance measurement, the projection measurement can reflect more information about the difference between two MVNSs. Furthermore, TODIM method, which considers the risk preferences of decision-makers, is significant in solving MCDM problems. In this study, we defined an improved comparison method, the projection and normalized projection measurements, and the projection-based difference measurement for MVNSs. Moreover, a novel MCDM method was established by incorporating the projection-based difference measurement with the fuzzy TODIM method. The proposed projection-based TODIM method was verified to be applicable and feasible by a numerical example of personnel selection and a comparative analysis. In addition, we discussed the influence of the parameter $t$.

The contribution of this study can be concluded as follows. First, this study utilized MVNSs to depict the fuzzy and hesitant information in the personnel selection processes. Second, an improved comparison method of MVNSs was defined to cover the defect of the extant comparison method. Third, the projection and normalized projection measurements were extended to multi-valued neutrosophic environments. Fourth, we presented a projection-based difference measurement of MVNSs based on the proposed projection and normalized projection measurements. Fifth, the projection measurement was combined with TODIM method and a projection-based TODIM method was constructed. The projection-based TODIM method more fully considers the difference between MVNSs than the fuzzy TODIM with the distance measurement.

There are several directions for future research. Firstly, this study utilizes the projection-based TODIM method in the personnel selection. In addition to the personnel selection, MCDM problems in a variety of other fields can be addressed with the projection-based TODIM method, 
including medical diagnosis, the selection of supplier, and the selection of renewable energy. Secondly, this study considers the risk preferences of decision-makers, while the interrelationships among criteria are ignored. In our future research, the method will be improved to cover this deficiency. Thirdly, in our numerical example, three criteria are taken for example. However, in practical application, many other criteria should be considered. A comprehensive framework for the personnel selection problems including all relevant criteria should be constructed on the basis of prior studies and the proposed personnel selection method in future research.

Acknowledgments This work was supported by the National Natural Science Foundation of China (Nos. 71501192 and 71571193).

\section{Compliance with ethical standards}

Conflict of interest The authors declare that there is no conflict of interest regarding the publication of this paper.

\section{References}

1. Alguliyev RM, Aliguliyev RM, Mahmudova RS (2015) Multicriteria personnel selection by the modified fuzzy VIKOR method. Sci World J. doi:10.1155/2015/612767

2. Liu HC, Qin JT, Mao LX, Zhang ZY (2015) Personnel selection using interval 2-tuple linguistic VIKOR method. Hum Factors Ergon Manuf Serv Ind 25(3):370-384

3. Dursun M, Karsak EE (2010) A fuzzy MCDM approach for personnel selection. Expert Syst Appl 37(6):4324-4330

4. Afshari RA, Nikolić M, Ćoćkalo D (2014) Applications of fuzzy decision making for personnel selection problem: a review. J Eng Manag Compet 4(2):68-77

5. Zhang S-F, Liu S-Y (2011) A GRA-based intuitionistic fuzzy multi-criteria group decision making method for personnel selection. Expert Syst Appl 38(9):11401-11405

6. Sang X, Liu X, Qin J (2015) An analytical solution to fuzzy TOPSIS and its application in personnel selection for knowledgeintensive enterprise. Appl Soft Comput 30:190-204

7. Zadeh LA (1965) Fuzzy sets. Inf Control 8(3):338-353

8. Zhou H, Wang J-Q, Zhang H-Y (2015) Grey stochastic multicriteria decision-making based on regret theory and TOPSIS. Int $\mathrm{J}$ Mach Learn Cybern. doi:10.1007/s13042-015-0459-x

9. Wang C, Wang J (2016) A multi-criteria decision-making method based on triangular intuitionistic fuzzy preference information. Intell Autom Soft Comput 22(3):473-482

10. Atanassov KT (1986) Intuitionistic fuzzy sets. Fuzzy Sets Syst 20(1):87-96

11. Atanassov K, Gargov G (1989) Interval valued intuitionistic fuzzy sets. Fuzzy Sets Syst 31(3):343-349

12. Torra V (2010) Hesitant fuzzy sets. Int J Intell Syst 25(6):529-539

13. Smarandache F (1998) Neutrosophy: neutrosophic probability, set, and logic. American Research Press, Rehoboth, pp 1-105

14. Smarandache F (1999) A unifying field in logics: neutrosophic logic. Neutrosophy, neutrosophic set, probability. American Research Press, Rehoboth, pp 1-141

15. Wang H, Smarandache F, Zhang Y, Sunderraman R (2010) Single valued neutrosophic sets. Multispace Multistruct 4:410-413
16. Tian Z, Wang J, Wang J, Zhang H (2016) A likelihood-based qualitative flexible approach with hesitant fuzzy linguistic information. Cognit Comput. doi:10.1007/s12559-016-9400-1

17. Zhou H, Wang J-Q, Zhang H-Y (2016) Multi-criteria decisionmaking approaches based on distance measures for linguistic hesitant fuzzy sets. J Oper Res Soc. doi:10.1057/jors.2016.41

18. Wu X, Wang J, Peng J, Chen X (2016) Cross-entropy and prioritized aggregation operator with simplified neutrosophic sets and their application in multi-criteria decision-making problems. Int J Fuzzy Syst. doi:10.1007/s40815-016-0180-2

19. Ye J (2014) A multicriteria decision-making method using aggregation operators for simplified neutrosophic sets. J Intell Fuzzy Syst 26(5):2459-2466

20. Tian ZP, Zhang HY, Wang J, Wang JQ, Chen XH (2016) Multicriteria decision-making method based on a cross-entropy with interval neutrosophic sets. Int J Syst Sci 47(15):3598-3608

21. Zhang H, Ji P, Wang J, Chen X (2015) An improved weighted correlation coefficient based on integrated weight for interval neutrosophic sets and its application in multi-criteria decision making problems. Int J Comput Intell Syst 8(6):1027-1043

22. Zhang H, Wang J, Chen X (2016) An outranking approach for multi-criteria decision-making problems with interval-valued neutrosophic sets. Neural Comput Appl 27(3):615-627

23. Broumi S, Talea M, Bakali A, Smarandache F (2016) Single valued neutrosophic graphs. J New Theory 10:86-101

24. Broumi S, Talea M, Bakali A, Smarandache F (2016) On bipolar single valued neutrosophic graphs. J New Theory 11:84-102

25. Broumi S, Smarandache F, Talea M, Bakali A (2016) An introduction to bipolar single valued neutrosophic graph theory. Appl Mech Mater 841:184-191

26. Broumi S, Bakali A, Talea M, Smarandache F (2016) Isolated single valued neutrosophic graphs. Neutrosophic Sets Syst 11:74-78

27. Broumi S, Talea M, Smarandache F, Bakali A (2016) Single valued neutrosophic graphs: degree, order and size. In: IEEE world congress on computational intelligence (accepted)

28. Zhou H, Wang J-q, Zhang H-y (2016) Grey stochastic multicriteria decision-making approach based on prospect theory and distance measures (in press)

29. Tian Z, Wang J, Wang J, Chen X (2015) Multi-criteria decisionmaking approach based on gray linguistic weighted Bonferroni mean operator. Int Trans Oper Res. doi:10.1111/itor.12220

30. Zhang H, Ji P, Wang J, Chen X (2016) A neutrosophic normal cloud and its application in decision-making. Cognit Comput. doi:10.1007/s12559-016-9394-8

31. Tian Z-P, Wang J, Wang J-Q, Zhang H-Y (2016) An improved MULTIMOORA approach for multi-criteria decision-making based on interdependent inputs of simplified neutrosophic linguistic information. Neural Comput Appl. doi:10.1007/s00521$016-2378-5$

32. Tian Z, Wang J, Wang J, Zhang H (2016) Simplified neutrosophic linguistic multi-criteria group decision-making approach to green product development. Group Decis Negot. doi:10.1007/ s10726-016-9479-5

33. Tian Z, Wang J, Zhang H, Wang J (2016) Multi-criteria decisionmaking based on generalized prioritized aggregation operators under simplified neutrosophic uncertain linguistic environment. Int J Mach Learn Cybern. doi:10.1007/s13042-016-0552-9

34. Ma Y, Wang J, Wang J, Wu X (2016) An interval neutrosophic linguistic multi-criteria group decision-making method and its application in selecting medical treatment options. Neural Comput Appl. doi:10.1007/s00521-016-2203-1

35. Ye J, Fu J (2016) Multi-period medical diagnosis method using a single valued neutrosophic similarity measure based on tangent function. Comput Methods Programs Biomed 123:142-149 
36. Ma H, Hu Z, Li K, Zhang H (2016) Toward trustworthy cloud service selection: a time-aware approach using interval neutrosophic set. J Parallel Distrib Comput 96:75-94

37. Karsak EE, Dursun M (2015) An integrated fuzzy MCDM approach for supplier evaluation and selection. Comput Ind Eng 82:82-93

38. Şahin R, Yiğider M (2014) A multi-criteria neutrosophic group decision making method based TOPSIS for supplier selection. arXiv preprint arXiv: 1412.5077

39. Wang JQ, Li XE (2015) TODIM method with multi-valued neutrosophic sets. Control Decis 30(6):1139-1142

40. Peng JJ, Wang JQ, Wu XH, Wang J, Chen XH (2015) Multivalued neutrosophic sets and power aggregation operators with their applications in multi-criteria group decision-making problems. Int J Comput Intell Syst 8(2):345-363

41. Ye J (2015) Multiple-attribute decision-making method under a single-valued neutrosophic hesitant fuzzy environment. J Intell Syst 24(1):23-36

42. Şahin R, Liu P (2016) Correlation coefficient of single-valued neutrosophic hesitant fuzzy sets and its applications in decision making. Neural Comput Appl. doi:10.1007/s00521-015-2163-x

43. Liu P, Zhang L (2015) The extended VIKOR method for multiple criteria decision making problem based on neutrosophic hesitant fuzzy set. arXiv preprint. arXiv:1512.0139

44. Zeng S, Baležentis T, Chen J, Luo G (2013) A projection method for multiple attribute group decision making with intuitionistic fuzzy information. Informatica 24(3):485-503

45. Yue Z (2013) An intuitionistic fuzzy projection-based approach for partner selection. Appl Math Model 37(23):9538-9551

46. Xu Z, Hu H (2010) Projection models for intuitionistic fuzzy multiple attribute decision making. Int J Inf Technol Decis Mak 9(02):267-280

47. Zhang X, Jin F, Liu P (2013) A grey relational projection method for multi-attribute decision making based on intuitionistic trapezoidal fuzzy number. Appl Math Model 37(5):3467-3477

48. Gomes L, Lima M (1992) TODIM: basics and application to multicriteria ranking of projects with environmental impacts. Found Comput Decis Sci 16(4):113-127

49. Gomes L, Lima M (1992) From modeling individual preferences to multicriteria ranking of discrete alternatives: a look at prospect theory and the additive difference model. Found Comput Decis Sci 17(3):171-184

50. Kahneman D, Tversky A (1979) Prospect theory: an analysis of decision under risk. Econom J Econom Soc 47(2):263-292

51. Krohling RA, Souza TTMd (2012) Combining prospect theory and fuzzy numbers to multi-criteria decision making. Expert Syst Appl 39(13):11487-11493

52. Liu P, Teng F (2014) An extended TODIM method for multiple attribute group decision-making based on 2-dimension uncertain linguistic variable. Complexity 21(5):20-30

53. Tosun Ö, Akyüz G (2015) A fuzzy TODIM approach for the supplier selection problem. Int J Comput Intell Syst 8(2):317-329

54. Gomes LFAM, Machado MAS, da Costa FF, Rangel LAD (2013) Criteria interactions in multiple criteria decision aiding: a Choquet formulation for the TODIM method. Procedia Comput Sci 17:324-331

55. Tseng M-L, Lin Y-H, Tan K, Chen R-H, Chen Y-H (2014) Using TODIM to evaluate green supply chain practices under uncertainty. Appl Math Model 38(11):2983-2995

56. Lourenzutti R, Krohling RA (2013) A study of TODIM in a intuitionistic fuzzy and random environment. Expert Syst Appl 40(16):6459-6468

57. Li Y, Shan Y, Liu P (2015) An extended TODIM method for group decision making with the interval intuitionistic fuzzy sets. Math Probl Eng. doi:10.1155/2015/672140

58. Zhang X, Xu Z (2014) The TODIM analysis approach based on novel measured functions under hesitant fuzzy environment. Knowl-Based Syst 61:48-58

59. Zhang M, Liu P, Shi L (2016) An extended multiple attribute group decision-making TODIM method based on the neutrosophic numbers. J Intell Fuzzy Syst 30(3):1773-1781

60. Yue Z, Jia Y (2015) A direct projection-based group decisionmaking methodology with crisp values and interval data. Soft Comput. doi:10.1007/s00500-015-1953-5

61. Qin J, Liu X, Pedrycz W (2016) Frank aggregation operators and their application to hesitant fuzzy multiple attribute decision making. Appl Soft Comput 41:428-452

62. Gomes LFAM (2009) An application of the TODIM method to the multicriteria rental evaluation of residential properties. Eur $\mathbf{J}$ Oper Res 193(1):204-211 